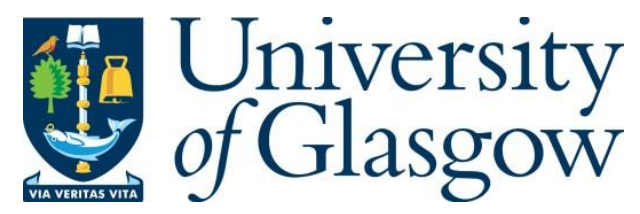

Parkinson, J. (2020) Investigating Spatial Skills in Computing Education. In: 2020 ACM Conference on International Computing Education Research (ICER '20), Dunedin, New Zealand, 11-14 Aug 2020, pp. 340-341. ISBN 9781450370929 (doi: $\underline{10.1145 / 3372782.3407109)}$ ).

There may be differences between this version and the published version. You are advised to consult the publisher's version if you wish to cite from it.

(C) Association for Computing Machinery 2020. This is the author's version of the work. It is posted here for your personal use. Not for redistribution. The definitive Version of Record was published in Proceedings of the 2020 ACM Conference on International Computing Education Research (ICER '20), Dunedin, New Zealand, 11-14 Aug 2020, pp. 340-341. ISBN 9781450370929.

http://eprints.gla.ac.uk/223928/

Deposited on: 14 October 2020

Enlighten - Research publications by members of the University of Glasgow http://eprints.gla.ac.uk 


\title{
Investigating Spatial Skills in Computing Education
}

\author{
Jack Parkinson \\ jack.parkinson@glasgow.ac.uk \\ University of Glasgow
}

\begin{abstract}
There is an intriguing connection between spatial skills and CS: those with better spatial skills tend to do better in many CS related tasks. Since spatial skills are malleable, it is tempting to simply introduce spatial skills training courses to students who are struggling and expect positive outcomes. While improved outcomes are being observed, it would be preemptive to introduce such schemes widely without better understanding the relationship. We do not know why spatial skills are important in CS, so while one might take the gains observed at face value, we stand to lose valuable insights into not only the abstract cognition involved in spatial skills which appears to be of value across STEM, but also reflective and nuanced understanding of how people engage with CS education.
\end{abstract}

\section{CCS CONCEPTS}

\section{- Social and professional topics $\rightarrow$ Computing education.}

\section{KEYWORDS}

computing education research; spatial skills

\section{BACKGROUND}

Over 60 years ago the first definite link between spatial skills and success in STEM was published [10]. More studies have explored this association, pointing to high spatial skills being correlated with a wide range of success metrics across STEM domains. Despite their reach and impact, little work was been done to introduce spatial skills training as a dedicated part of STEM teaching; even though they can be trained in a range of ways across divides of age, gender, academic attainment and socio-economic status [11].

While many spatial skills to STEM studies are correlational, Sorby has shown over decades that training spatial skills of incoming engineering students improves retention and GPAs [9], even in electives [8]. Two new studies have demonstrated the causal link between training spatial skills and improved CS outcomes: students with initially poor spatial skills and computing outcomes were able to improve in both after a spatial skills intervention $[1,6]$.

There is also evidence that the relationship is bi-directional, and STEM learning can improve spatial skills over time. Pallrand \& Seeber identified that a cohort of Physics students improved their spatial ability over a physics course while a cohort of liberal arts students did not [4]. Work in CS indicates that those who progress further in academic careers have higher spatial skills (though this may be due to a rising mean as less spatially skilled people leave) [5]

The research to date tells a compelling story: high spatial ability and success in STEM/CS are linked. The nature of this relationship is not well understood, with only a few largely untested theories being proposed [3,5], but some relationship is evident. This is of interest because spatial skills training can be conducted with concepts which we inherently understand by living in a physical world (eg. rotation and 3D space) making it accessible at a low resource cost compared to domain specific introductory programmes.

\section{MOTIVATION}

We can confidently state that spatial skills and spatial skills training are of value in CS education (CS ed), but without understanding the relationship we cannot state how or why they have an impact. As such, we cannot be sure that typical spatial skills training programmes are maximally effective in developing the skills which are applicable in CS or what long term effects they might have.

I propose that there exists a set of abstract skills utilised by both spatial and CS exercises [5]. These abstract skills (eg. mental model building) can be developed by spatial skills training or CSE, which lines up with observations in physics [4]. However, in order to construct mental models of concepts in CS, such as notional machines [2], a grasp of contextual, domain specific knowledge is required. This needn't be the case in spatial skills training, where the construction of a mental model requires only the formulation of a 3D internal structure from 2D representations; not necessarily a trivial task, but one that has limited domain knowledge cost, since we are all aware of the nature of 3D space and 2D representations.

So while spatial skills training may be a fast, effective route to developing these skills, we don't have a clear indication of how relevant it is as a whole. The goal of this research is to identify the abstract skills existing between spatial skills and CS so that they can be clearly examined and developed, possibly without the need for a spatial context. Exemplifying mental model building: if we can identify this as an abstract skill used in both spatial and CS tasks, we may be able to develop it in either side with the correct approach - meaning that CS exercises can train these skills without needing to dedicate resources to independent training.

\section{RESEARCH STATEMENT AND HYPOTHESES}

My research statement is that spatial skills may or may not be exceptionally important in CSE, but we can use them as a lens to expose and explore CS skills. I want to explore the interaction between spatial skills and CS: spatial skills training is low-cost, so utilising it in CSE should be explored. But at another level, I want to explore CS skills and development through the lens of this phenomenon. Therefore I present the following hypotheses:

(1) A set of abstract, cognitive skills are required for spatial skills exercises and success in CS, explaining the documented relationship between the two.

(2) We can use our understanding of spatial skills to better understand CS skill sets.

Hypothesis 1 is about understanding underlying mechanisms; the threads which draw the two domains together. Hypothesis 2 is more exploratory and can potentially help us understand more 
about CSE. Eg. what cognitive factors cause one student to excel in CSE and while another struggles, when they have similar backgrounds and education? Can we develop these abstract skills independently of spatial skills training, as part of into CSE?

\section{RESEARCH GOALS, METHODS AND PROGRESS}

The intended research can be broken into five distinct bodies.

Showing that spatial skills training is of value through intervention. This is to determine clearly whether deeper exploration of the relationship is of value; it indicates that the relationship is causal, and can therefore be manipulated. It does not necessarily help to answer the overarching question of "why?" but does give us confidence that continued investigation is valuable.

This work has been completed, with positive results for introductory CS students [6]. The methodology replicated Sorby's engineering studies: incoming CS students were given a spatial skills test, and those scoring below $60 \%$ were required to take a training course. Bockmon et al. conducted a similar study simultaneously, which evidence for the value of spatial skills training in CS [1].

Associating spatial skills with low-level, precise CS exercises. This is to isolate the abstract skills involved in both sides of the relationship. In the first iteration of this work, a spatial skills test was closely analysed to determine what these skills could be. A test was developed to utilise a subset of the skills identified based on expression evaluation in Python rather than 3D mental rotation.

This work has been completed and submitted for publication, showing a strong correlation between participants' ability to complete both tests effectively. This doesn't prove that abstract skills identified are being put to use, but provides evidence for a cognitive relationship. Future work is tabled for late 2020 involving more facets on either side (orientation, debugging, closure speed, etc.)

Removing the spatial element. Since we know that Physics instruction can develop spatial skills and presumably the useful abstract skills, we should investigate whether a computing training course could do the same. By developing an introductory programming module constructed with an effort to develop the abstract skills identified in the previous research, we can measure if the spatial skills of participants are improved with pre- and post-tests. This will give an indication of whether, and how effectively, abstract skills can be trained through directed CS instruction as observed in spatial skills test scores. This work is scheduled for summer 2020.

Longitudinal analysis of spatial skills in CS majors. For the past three years I have been conducting spatial skills tests of growing groups of level 1 CS students, with $76 \%$ of the 2019 cohort tested in their first week of study. I expect $~ 100 \%$ testing rates for the $2020 / 21$ academic year, providing a broad basis to conduct a robust longitudinal study. With this cohort, we can answer questions about the nature of spatial skills in CSE. Will we observe that those with poor spatial skills drop out/change majors, or will the cohort's spatial skills improve on average over time? Do the high spatially skilled perform exceptionally in computing tasks across the board, even into Honours; are students with poor spatial skills able to progress? If so, how do their learning methods and techniques compare to their high spatially skilled peers?
This analysis will provide a better understanding of how students with varied spatial skills progress through CS. With closer analysis, e.g. re-testing, interviews, we can examine the way spatial skills interact with different aspects CS careers. Analysis of the 2017 CS0 intake, now one year into their two-year Major phase, is underway.

Examining spatial skills in other demographics. Most of the research connecting spatial skills and CS has been on university students. Margulieux indicates that spatial skills are likely of more benefit to novices than experts [3], however we don't know how this interacts with the idea that we are all novices at the learning edge when picking up new skills [7]: does this apply to people learning new languages or technologies? Investigating these theories would strengthen our understanding of how the cognitive relationship manifests, so I intend to develop a survey to be taken alongside a spatial skills test to expose an expert developer's thought process, problem approaches and learning techniques in relation to abstract skills and distributed around the industrial community. This work is currently conceptual and planned to be developed in early 2021 .

\section{REFERENCES}

[1] Ryan Bockmon, Stephen Cooper, William Koperski, Jonathan Gratch, Sheryl Sorby, and Mohsen Dorodchi. 2020. A CS1 Spatial Skills Intervention and the Impact on Introductory Programming Abilities. In Proceedings of the 51st ACM Technical Symposium on Computer Science Education. 766-772.

[2] Benedict Du Boulay. 1986. Some difficulties of learning to program. Fournal of Educational Computing Research 2, 1 (1986), 57-73.

[3] Lauren E Margulieux. 2019. Spatial Encoding Strategy Theory: The Relationship between Spatial Skill and STEM Achievement. In Proceedings of the 2019 ACM Conference on International Computing Education Research. 81-90. https://doi. org/10.1145/3291279.3339414

[4] George J Pallrand and Fred Seeber. 1984. Spatial ability and achievement in introductory physics. Fournal of Research in Science Teaching 21, 5 (1984), 507516.

[5] Jack Parkinson and Quintin Cutts. 2018. Investigating the Relationship Between Spatial Skills and Computer Science. In Proceedings of the 2018 ACM Conference on International Computing Education Research. ACM, 106-114. https://doi.org/ 10.1145/3230977.3230990

[6] Jack Parkinson and Quintin Cutts. 2020. The Effect of a Spatial Skills Training Course in Introductory Computing. http://eprints.gla.ac.uk/213969/

[7] Anthony Robins. 2010. Learning edge momentum: A new account of outcomes in CS1. Computer Science Education 20, 1 (2010), 37-71.

[8] Sheryl Sorby, Norma Veurink, and Scott Streiner. 2018. Does spatial skills instruction improve STEM outcomes? The answer is 'yes'. Learning and Individual Differences 67 (2018), 209-222. https://doi.org/10.1016/j.lindif.2018.09.001

[9] Sheryl A Sorby. 2009. Educational research in developing 3-D spatial skills for engineering students. International fournal of Science Education 31, 3 (2009), 459-480.

[10] Donald E Super and Paul B Bachrach. 1957. Scientific careers and vocational development theory: A review, a critique and some recommendations. (1957).

[11] David H Uttal, Nathaniel G Meadow, Elizabeth Tipton, Linda L Hand, Alison R Alden, Christopher Warren, and Nora S Newcombe. 2013. The malleability of spatial skills: A meta-analysis of training studies. 